\title{
Evaluation of Fever and Its Related Factors during 4 Weeks after Primary Total Hip Arthroplasty
}

\author{
Yoshinori Ishii ${ }^{1}$, Hideo Noguchi ${ }^{1}$, Mitsuhiro Takeda ${ }^{1}$, Junko Sato ${ }^{1}$, \\ Takeshi Yamamoto ${ }^{1}$, Yo-Ichiro Domae ${ }^{2}$ \\ ${ }^{1}$ Ishii Orthopaedic and Rehabilitation Clinic, Gyoda, Japan \\ ${ }^{2}$ Department of Orthopaedic Surgery, Shibata Prefectural Hospital, Shibata, Japan \\ Email: ishii@sakitama.or.jp
}

Received November 12, 2012; revised December 14, 2012; accepted December 23, 2012

\begin{abstract}
The purpose of this study was to describe the incidence and characteristics of postoperative fever $\left(\mathrm{POF} ; \geq 38^{\circ} \mathrm{C}\right)$ and clarify the correlation between POF and febrile-related factors during 4 weeks after primary total hip arthroplasty (THA). A total of 56 patients were included in the study (67 THAs). The preoperative diagnosis for all patients was osteoarthritis. The average follow-up period was 90 months. No patients developed deep infection during the follow-up period. The maximum mean peak daily temperature was $37.7^{\circ} \mathrm{C}$ on the day of the surgery and the next day. Fifty-two percent of patients experienced POF, with most developing a maximum temperature (MT) on the day of surgery and the latest occurring by postoperative day (POD) 3 . Only C-reactive protein $(\mathrm{R}=0.384, \mathrm{p}=0.001)$ among the febrile-related factors had a weak correlation with MT. Four hips showed a POF of $>38^{\circ} \mathrm{C}$ later than POD 7; all four of these patients were diagnosed with a urinary tract infection. POF is common and may be part of a normal inflammatory response to tissue injury after THA. More than half of patients may develop POF within 1 week, with most developing MT on POD 0 and the latest occurring by POD 3. In addition, urinary tract infections might be an initial reason for POF in the subsequent 3 weeks.
\end{abstract}

Keywords: Total Hip Arthroplasty; Post-Operative Fever; Maximum Temperature; C-Reactive Protein; Urinary Tract Infection

\section{Introduction}

A postoperative fever (POF), defined as a body temperature of $\geq 38^{\circ} \mathrm{C}\left(100.4^{\circ} \mathrm{F}\right)$ in the postoperative period, is experienced in $40 \%$ to $50 \%$ of patients who undergo total joint arthroplasty (TJA) [1]. Although fever is commonly associated with infection, POF may be a normal postoperative physiological febrile response. A rise in temperature may occur within the first 5 days following hip and knee replacement [1-5]. Most authors have concluded that a sepsis workup is unnecessary if the febrile response progressively decreases and that it should be based on physical examination or symptomatic findings. Conversely, infection after TJA presents a significant clinical and financial burden in terms of both diagnosis and treatment [5-7] of periprosthetic sepsis. A prompt diagnosis of periprosthetic infection in TJA permits early treatment, which is particularly important for infections that occur acutely within the first 6 weeks [7]. In addition, early acute infection is defined as infection that occurs in the first 4 weeks after surgery [8]. Gustilo et al. [8] recommended that surgical debridement and irrigation in total hip arthroplasty (THA) must be done immediately after a thorough clinical and radiographic evaluation and diagnosis of a septic hip. Therefore, it is very important for surgeons and primary care physicians who are used to assisting with management of medical problems affecting these patients to recognize the characteristics of POF in the early period after THA and properly judge the necessity of a further workup to identify the infection.

The Japanese national medical insurance system might allow patients to determine, to some degree, the length of their hospital stay if there are reasonable reasons to stay, such as remaining uncontrolled pain, unstable transfer, and other perioperative complications. In fact, Mitsuyasu et al. [9] reported that the mean length of stay was 50.78 \pm 30.34 days in high-volume hospitals and $70.61 \pm 40.34$ days in low-volume hospitals after THA in Japan. Because the average hospital stay was $40 \pm 18$ days after THA in our institute [10], we could investigate the various factors affecting POF and indicators of infection such as blood loss, transfusion, leukocytosis, and serum C-reactive protein (CRP) level during a longer period compared with previous studies. Most studies on POF after THA reported their findings from the immediate 
postoperative days (PODs) during patients' hospital stays [1-3]. The purpose of this study was to describe the incidence and characteristics of POF and clarify the correlation between POF and febrile-related factors for a longer period (4 weeks) than that in previous studies after THA surgery.

\section{Patients and Methods}

Institutional review board approval was obtained to retrospectively review medical records and analyze pertinent data. This study was a retrospective analysis of patients treated with primary THA from 1998 to 2011. A total of 56 patients were included in the study (67 THAs). The preoperative diagnosis for all patients was osteoarthritis. The average follow-up period was 90 months (range, 6 180 months). No patients developed deep infection during this period. The clinical characteristics of the patients are summarized in Table 1.

\subsection{Perioperative Treatment Protocol}

During surgery, $800 \mathrm{ml}$ of autogenous blood that had been preserved 1 and 2 weeks ( $400 \mathrm{ml}$ each) preoperatively was returned to the patient. A CBCII ConstaVac Blood Conservation System (Stryker, Kalamazoo, MI) was used in all patients after surgery. The volume of blood collected was returned to the patients within 6 hours of surgery. A foot pump (A-V Impulse System; Novamedix, Andover, UK) was used to prevent deep venous thrombosis. The drains were removed routinely during the first dressing change on POD 1. After drain removal, the patients were allowed full weight-bearing ambulation with a hip brace [10] to prevent initial hip dislocation. All patients received perioperative antibiotics for prophylaxis and analgesics for pain. No patients received medication for thromboembolism.

\subsection{Definitions}

All patients' charts were evaluated for POF for 4 weeks of their hospital stay. Axillary temperatures were recorded routinely using standard digital thermometers

Table 1. Patient characteristics.

\begin{tabular}{cc}
\hline Number of patients & 56 patients, $67 \mathrm{hips}$ \\
Mean age & $62 \pm 11$ years \\
Sex: male 7 (9 THA); female $49(58 \mathrm{THA})$ \\
Body height & $154 \pm 7 \mathrm{~cm}$ \\
Body weight & $56 \pm 10 \mathrm{~kg}$ \\
BMI & $24 \pm 3$ \\
Follow-up period (months) & $90 \pm 47$ \\
\hline
\end{tabular}

every hour on the day of surgery and then every 8 hours by staff following a specific nursing care map. The day of surgery started in their ward and continued until 6:00 AM on the following day. POD 1 began at 6:00 AM on the day after surgery and continued for 24 hours. Subsequent PODs began and ended at 6:00 AM in a repeating sequence. If a patient underwent bilateral procedures during different hospitalizations, each surgical procedure and postoperative course was analyzed separately (no simultaneous bilateral procedures were performed). Perioperative days were defined as reported by Summersell et al. [4] and modified as follows: the day before the operation was day 1 (POD 1), which was also called the preoperative baseline; the day of surgery was day 0 (POD 0); the first day after surgery was day 1 (POD 1); and so on. The peak daily temperature (PDT) was defined as the highest temperature recorded on a given day for each patient. The maximum temperature (MT) was the highest PDT recorded for each patient during the study period.

In patients who experienced fever, the MT and the PDT were appropriately recorded. Serological factors that reportedly affect the febrile response, such as white blood cell (WBC) count [11], hematocrit (Ht) [12], and CRP level, were routinely investigated to monitor for bacterial infection [13] and level of intraoperative tissue damage [14] at postoperative weeks 1 and 4. Leukocytosis was defined as a WBC count of $\geq 9000 / \mathrm{ml}$. Estimated blood loss was calculated using the maximum decrease in Ht levels measured 1 week after surgery and normalized to the patient's weight and height according to the method of Gross [15].

\subsection{Statistical Analysis}

Spearman's rank correlation coefficient was used to evaluate the relationships between the MT and POFrelated factors such as age, body mass index (BMI), operation time, estimated blood loss, autotransfusion volume, and serological results. The strength of the rank correlation coefficient was defined as follows: strong, 0.7 to 1.0 ; moderate, 0.4 to 0.7 ; and weak, 0.2 to 0.4 . Differences in the MT between men and women and between spinal and general anesthesia were compared using Wilcoxon's rank sum test. Statistical analyses were performed using IBM SPSS Statistics ver. 20 (IBM Japan, Tokyo, Japan). In all tests, a p-value of $<0.05$ was considered to indicate statistical significance.

\section{Results}

The maximum mean PDT during 4 weeks was $37.7^{\circ} \mathrm{C}$ on POD 0 and 1 , and slowly decreased toward $<37.0^{\circ} \mathrm{C}$ after POD 4. The highest number of patients with a POF occurred on POD 0 , followed by POD 1 . In addition, four 
hips showed a POF of $>38^{\circ} \mathrm{C}$ later than POD 7; all of these patients were diagnosed with a urinary tract infection (Table 2). The average time points showing the MT on POD 0 were $8 \pm 4$ hours after surgery and $4 \pm 4$ hours after return of autogenous blood, respectively.

Of 67 hips, 35 (52\%) showed a POF. Most patients developed MT on POD 0 followed by POD 1 (Table 3).

Only CRP ( $\mathrm{R}=0.384, \mathrm{p}=0.001)$ among febrile related factors had a weak correlation with MT (Table 4). In addition, there were no significant differences in MT between men and women $(\mathrm{p}=0.285)$, nor was there a significant correlation with anesthesia $(p=0.2687)$.

No patients showed leukocytosis during the 4 weeks. Ht was lowest and CRP was highest in the first week postoperatively. They gradually approached preopera-

Table 2. Mean peak daily temperature during follow-up period.

\begin{tabular}{ccc}
\hline POD & Mean PDT (S.D.) & No. of $\geq 38^{\circ} \mathrm{C}$ \\
\hline-1 & $36.2(0.4)$ & 0 \\
0 & $37.7(0.6)$ & 24 \\
1 & $37.7(0.7)$ & 19 \\
2 & $37.3(0.7)$ & 10 \\
3 & $37.0(0.6)$ & 2 \\
4 & $36.8(0.6)$ & 1 \\
5 & $36.6(0.6)$ & 1 \\
6 & $36.5(0.5)$ & 1 \\
7 & $36.4(0.6)$ & 0 \\
$-14^{*}$ & $36.9(0.3)$ & 2 \\
$-21^{* *}$ & $36.8(0.4)$ & 0 \\
$-28^{\#}$ & $36.7(0.5)$ & 2 \\
\hline
\end{tabular}

POD: postoperative day; PDT: peak daily temperature. Mean PDT of each patient during each week: ${ }^{*} \mathrm{POD} 8$ to $14 ;{ }^{* *} \mathrm{POD} 15$ to $21 ;{ }^{*} \mathrm{POD} 22$ to 28.

Table 3. Postoperative day of development of maximum temperature.

\begin{tabular}{ccc}
\hline POD & Day of MT & No. of $\geq 38^{\circ} \mathrm{C}$ in MT \\
\hline-1 & 0 & 0 \\
0 & 31 & 18 \\
1 & 28 & 13 \\
2 & 7 & 4 \\
3 & 1 & 0 \\
4 & 0 & 0 \\
5 & 0 & 0 \\
6 & 0 & 0 \\
7 & 0 & 0 \\
\hline
\end{tabular}

No.: number; POD: postoperative day; MT: maximum temperature. tive levels and stabilized during the subsequent 3 weeks after surgery (Table 5).

\section{Discussion}

The most important finding of this study was that $52 \%$ of patients who underwent THA experienced POF, with most developing MT on POD 0 and the latest occurring by POD 3. POF may occur until as late as POD 6 . The rate of POF was higher and the POD of MT was earlier than those of recent reports from the US [1,2,5]. Approximately $36 \%$ [1] and $23.1 \%$ [2] of patients who undergo THA develop a fever, with most developing an MT on POD 2 [1] or POD 2 or 3 [5]. Previous reports from Australia also revealed that $62.5 \%$ of POFs reached $38^{\circ} \mathrm{C}$ [4] and that an MT occurred in most patients on POD 1 [3,4], gradually leveling off toward a normal temperature by POD 5 . In addition, after 1 week, two patients revealed a POF due to a urinary tract infection in the present study. For 4 weeks after surgery in the present study, there were no correlations between MT and serological factors $[2,12,16]$ with the exception of CRP or between MT and BMI, MT and gender, MT and age, or MT and anesthesia.

Our study has some limitations. First, it was a retrospective analysis and involved a relatively small number of patients. Second, the patients in this study were treated with non-steroidal anti-inflammatory drugs (NSAIDs) for pain control. The NSAIDs could have blunted the febrile

Table 4. Correlation between maximum temperature and postoperative fever-related factors by Spearman's rank correlation coefficient.

\begin{tabular}{ccc}
\hline & $\mathrm{R}$ & $\mathrm{p}$ \\
\hline Age & -0.203 & 0.099 \\
BMI & -0.086 & 0.487 \\
Operation Time & 0.204 & 0.098 \\
Estimate blood loss & -0.097 & 0.437 \\
Auto-transfusion & -0.108 & 0.383 \\
White blood cell count & 0.102 & 0.413 \\
Hematcrit ${ }^{\#}(\%)$ & -0.152 & 0.219 \\
C-reactive protein & $0.384^{*}$ & $0.001^{*}$ \\
\hline
\end{tabular}

"Postoperative 1 week; BMI: body mass index.

Table 5. Changes of serological investigations during 4 weeks after surgery.

\begin{tabular}{cccc}
\hline Mean (S.D.) & Preoperative & PO $1 \mathrm{w}$ & PO 4 w \\
\hline WBC count & $5813(1380)$ & $5482(1018)$ & $5079(1249)$ \\
Ht (\%) & $40.9(3.7)$ & $33.1(4.4)$ & $37.8(4.0)$ \\
CRP & $0.34(0.74)$ & $2.09(1.58)$ & $0.42(1.46)$ \\
\hline
\end{tabular}

S.D.: standard deviation; PO: postoperative; WBC: white blood cell; $\mathrm{Ht}$ : hematocrit; CRP: C-reactive protein $(\mathrm{mg} / \mathrm{l})$. 
response in these patients. Third, all patients received antibiotics from POD 0 to 3 . The antibiotics may have also blunted the febrile response. Fourth, although we recognized the release [17] or increase [11] of pyogenic mediators such as interleukin (IL)-6, IL-1 $\beta$, and tumor necrosis factor $\alpha$ in both the serum and drainage of patients who underwent TJA, the autogenous blood collected with the Blood Conservation System was returned to the patients within 6 hours of surgery. We did not evaluate their pyogenic mediators because of cost-effectiveness and difficulty during busy practical performance. A strength of this study is that the fever pattern was analyzed during a longer period (4 weeks) than that in previous studies [1-5] after THA.

Fortunately, no patients developed deep infection after THA during the average follow-up period of 90 months in the current study. However, with regard to the correlation between POF and infection, Ward et al. [5] reported that fever occurring after POD 3 and multiple febrile days are independent predictors of a positive workup, and patients with an $\mathrm{MT}$ of $\geq 39.0^{\circ} \mathrm{C}$ had a significantly higher rate of positive fever evaluations. The current study might support their results. The MT in the current study also occurred until POD 3 and POF until POD 2. Taking these results into account, a 2- or 3-day hospital stay as reported by the most recent US studies $[18,19]$ might be reasonable. This is because POF may be recognized as an infection-related fever when MT occurs after POD 3 and as a tissue-destruction-related fever when MT occurs until POD 3 after THA.

With regards to serological investigations (WBC count, $\mathrm{Ht}$, and CRP level), the CRP level at 1 week after surgery revealed a weak correlation with MT. The CRP level increases and peaks in 2 to 3 days followed by a rapid fall [14] after a major operation, including THA. Thus, considering the high mean PDT in this study, we could expect a strong correlation between CRP and MT if the CRP level had been investigated at 2 to 3 days after surgery. CRP levels reportedly rise after infection and tissue destruction. CRP might be a valuable supplement in the monitoring of infection after THA [20]. Deep periprosthetic infection must be suspected if the WBC count, erythrocyte sedimentation rate, or CRP value is elevated [21]. White et al. [13] stated that rising CRP levels after POD 3 may indicate a complication of THA and total knee arthroplasty (TKA), such as infection. The combination of CRP and IL- 6 was recently shown to be as useful as intraoperative frozen section for the detection of periprosthetic infection [22]. Therefore, because no patients developed deep infection in this series, the higher CRP level may have been induced by soft tissue destruction or reaming of the acetabulum and/or femoral stem for a long period of time. Although it was difficult to confirm the degree of tissue destruction in detail from the medical charts, it is reasonable to speculate that a larger amount of tissue injury was induced during surgery involving a longer operation time in patients with a higher CRP level. Frank et al. [23] previously reported longer operation time as one of most closely correlated factors to core body temperature.

The correlation between transfusion and POF after TJA is a controversial issue. Kennedy et al. [12] identified a higher body temperature in patients who underwent TKA and received a homologous blood transfusion. Furthermore, occasional febrile reactions during autologous retransfusion of postoperatively collected drainage blood are reportedly only side effects [24]. A relationship was shown between increased IL-6 concentrations (not volume) in shed drainage blood and the occurrence of febrile reactions after retransfusion of such blood [17]. However, Shaw and Chung [3] reported similar rates with and without transfusion. Andres et al. [11] stated that transfusion administration is not associated with fever. We also did not reveal a significant correlation between autologous retransfusion volume and febrile reaction.

In addition, the correlation between blood loss and POF is a controversial issue. No significant differences were identified between patients with an estimated blood loss of $<500 \mathrm{ml}$ and patients with an estimated blood loss of $>500 \mathrm{ml}$ [3]. Andrew et al. [11] reported that the blood loss or drainage output amounts are not associated with fever. In contrast, elevation in body temperature can be inversely correlated with a drop in $\mathrm{Ht}$ in patients who have undergone TKA [12]. We did not find a statistical correlation between estimated blood loss and febrile reaction.

Fevers may be due to septic and aseptic causes such as pulmonary embolism, pneumonia, urinary tract infection, deep venous thrombosis, surgical wound infection, and intravenous phlebitis [25]. POF has been linked to a transient disturbance of temperature regulation after general anesthesia, surgery, and pyrogens (both endogenous and exogenous) acting on the nervous system [25]. It is very important for surgeons and primary care physicians who are used to assisting with the management of medical problems affecting these patients to properly judge the necessity of a further workup to identify the infection. The results of the current study might help surgeons and primary care physicians to recognize the characteristics of POF in the first 4 weeks after THA that might play an important role in distinguishing POF from infectionrelated fever as well as recognizing physical examination or symptomatic findings. In addition, understanding the results of the current study can help surgeons to reduce unnecessary sepsis workups and prevent possible delays in discharge, and patients may confine their visits to primary care physicians to the first 4 weeks after surgery 
when they are determined to have an early acute infection [8]. As a result, the overall cost of THA could be lower.

In conclusion, POF is common and may be part of a normal inflammatory response to tissue injury after THA. More than half of patients may experience POF within 1 week, with most developing an MT on POD 0 and the latest occurring by POD 3 during the first week postoperatively. In addition, urinary tract infections might be an initial reason for the development of POF during the subsequent 3 weeks.

\section{REFERENCES}

[1] C. Athanassious, A. Samad, A. Avery, J. Cohen and D. Chalnick, "Evaluation of Fever in the Immediate Postoperative Period in Patients Who Underwent Total Joint Arthroplasty," Journal of Arthroplasty, Vol. 26, No. 8, 2011, pp. 1404-1408. doi:10.1016/j.arth.2011.02.019

[2] A. P. Czaplicki, J. E. Borger, J. R. Politi, B. T. Chambers and B. C. Taylor, "Evaluation of Postoperative Fever and Leukocytosis in Patients after Total Hip and Knee Arthroplasty," Journal of Arthroplasty, Vol. 26, No. 8, 2011, pp. 1387-1389. doi:10.1016/j.arth.2010.12.024

[3] J. A. Shaw and R. Chung, "Febrile Response after Knee and Hip Arthroplasty," Clinical Orthopaedics and Related Research, Vol. 367, 1999, pp. 181-189. doi:10.1097/00003086-199910000-00022

[4] P. C. Summersell, A. Turnbull, G. Long, et al., "Temperature Trends in Total Hip Arthroplasty: A Retrospective Study," Journal of Arthroplasty, Vol. 18, No. 4, 2003, pp. 426-429. doi:10.1016/S0883-5403(03)00015-9

[5] D. T. Ward, E. N. Hansen, S. K. Takemoto and K. J. Bozic, "Cost and Effectiveness of Postoperative Fever Diagnostic Evaluation in Total Joint Arthroplasty Patients," Journal of Arthroplasty, Vol. 25, No. 6, 2010, pp. 43-48.

[6] K. J. Bozic and M. D. Ries, "The Impact of Infection after Total Hip Arthroplasty on Hospital and Surgeon Resource Utilization," Journal of Bone and Joint Surgery, Vol. 87, No. 8, 2005, pp. 1746-1751. doi:10.2106/JBJS.D.02937

[7] A. D. Toms, D. Davidson, B. A. Masri and C. P. Duncan, "The Management of Peri-Prosthetic Infection in Total Joint Arthroplasty," Journal of Bone and Joint Surgery, Vol. 88, No. 2, 2006, pp. 149-155. doi:10.1302/0301-620X.88B2.17058

[8] R. B. Gustilo and L. A. C. Leagogo, "Management of Infected Total Hip Replacement. Orthopaedic Infection. Diagnosis and Treatment," WB Saunders, Philadelphia, 1989, pp. 224-233.

[9] S. Mitsuyasu, A. Hagihara, H. Horiguchi and K. Nobutomo, "Relationship between Total Arthroplasty Case Volume and Patient Outcome in an Acute Care Payment System in Japan," Journal of Arthroplasty, Vol. 21, No. 5, 2006, pp. 656-663. doi:10.1016/j.arth.2005.09.007

[10] Y. Ishii, H. Noguchi, M. Takeda, J. Sato and Y. Domae, "Efficacy of an Abduction Brace in Preventing Initial Dislocation in the Early Postoperative Period after Pri- mary Total Hip Arthroplasty," Surgical Science, Vol. 3, No. 3, 2012, pp. 131-135. doi:10.4236/ss.2012.33026

[11] B. M. Andres, D. D. Taub, I. Gurkan and J. F. Wenz, "Postoperative Fever after Total Knee Arthroplasty: The Role of Cytokines," Clinical Orthopaedics and Related Research, Vol. 415, 2003, pp. 221-231. doi:10.1097/01.blo.0000093914.26658.55

[12] J. G. Kennedy, W. B. Rodgers, D. Zurakowski, et al., "Pyrexia after Total Knee Replacement. A Cause for Concern?" American Journal of Orthopaedics, Vol. 26, No. 8, 1997, pp. 549-552,554.

[13] J. White, M. Kelly and R. Dunsmuir, "C-Reactive Protein Level after Total Hip and Total Knee Replacement," Journal of Bone and Joint Surgery, Vol. 80, No. 5, 1998, pp. 909-911. doi:10.1302/0301-620X.80B5.8708

[14] R. O. Niskanen, O. Korkala and H. Pammo, "Serum CReactive Protein Levels after Total Hip and Knee Arthroplasty," Journal of Bone and Joint Surgery, Vol. 78, No. 3, 1996, pp. 431-433.

[15] J. B. Gross, "Estimating Allowable Blood Loss: Corrected for Dilution," Anesthesiology, Vol. 58, No. 3, 1983, pp. 277-280. doi:10.1097/00000542-198303000-00016

[16] D. Schey, E. M. Salom, A. Papadia and M. Penalver, "Extensive Fever Workup Produces Low Yield in Determining Infectious Etiology," American Journal of Obstetrics and Gynecology, Vol. 192, No. 5, 2005, pp. 17291734.

[17] M. Handel, J. Winkler, R. F. Hörnlein, et al., "Increased Interleukin-6 in Collected Drainage Blood after Total Knee Arthroplasty: An Association with Febrile Reactions during Retransfusion," Acta Orthopaedica Scandinavica, Vol. 72, No. 3, 2001, pp. 270-272. doi: $10.1080 / 00016470152846600$

[18] A. F. Chen, M. K. Stewart, A. E. Hey and B. A. Klatt, "Effect of Immediate Postoperative Physical Therapy on Length of Stay for Total Joint Arthroplasty Patients," Journal of Arthroplasty, Vol. 27, No. 6, 2012, pp. 851856. doi:10.1016/j.arth.2012.01.011

[19] K. Juliano, D. Edwards, D. Spinello, et al., "Initiating Physical Therapy on the Day of Surgery Decreases Length of Stay without Compromising Functional Outcomes Following Total Hip Arthroplasty," The Musculoskeletal Journal of Hospital for Special Surgery, Vol. 7, No. 1, 2011, pp. 16-20.

[20] L. Sanzén and Å. S. Carlsson, "The Diagnostic Value of C-Reactive Protein in Infected Total Hip Arthroplasties," Journal of Bone Joint Surgery, Vol. 71, No. 4, 1989, pp. 638-641.

[21] C. J. Della Valle, J. D. Zuckerman and P. E. Di Cesare, "Periprosthetic Sepsis," Clinical Orthopaedic and Related Research, Vol. 420, 2004, pp. 26-31. doi:10.1097/00003086-200403000-00005

[22] M. A. Buttaro, I. Tanoira, F. Comba and F. Piccaluga, "Combining C-Reactive Protein and Interleukin-6 May Be Useful to Detect Periprosthetic Hip Infection," Clinical Orthopaedic and Related Research, Vol. 468, No. 12, 2010, pp. 3263-3267. doi:10.1007/s11999-010-1451-0

[23] S. M. Frank, M. J. Kluger and S. L. Kunkel, "Elevated 
Thermostatic Setpoint in Postoperative Patients," Anesthesiology, Vol. 93, No. 6, 2000, pp. 1426-1431.

doi:10.1097/00000542-200012000-00014

[24] P. M. Faris, M. A. Ritter, E. M. Keating and C. R. Valer, "Unwashed Filtered Shed Blood Collected after Knee and Hip Arthroplasties," Journal of Bone Joint Surgery, Vol.
73, No. 8, 1991, pp. 1169-1178.

[25] S. Kenan, M. Liebergall, E. Simchen and S. Porat, "Fever Following Orthopedic Operations in Children," Journal of Pediatric Orthopaedics, Vol. 6, No. 2, 1986, pp. 139142. doi:10.1097/01241398-198603000-00003 